Genetics Research

cambridge.org/grh

\section{Short Paper}

Cite this article: Mesoraca A et al (2020). Cellfree DNA screening for aneuploidies in 7113 pregnancies: single Italian centre study. Genetics Research 102, e5, 1-4. https://doi.org/ $10.1017 /$ S001667232000004X

Received: 14 January 2020

Revised: 22 April 2020

Accepted: 27 April 2020

\section{Keywords:}

cell-free foetal DNA; cffDNA; chromosome aneuploidies; next-generation sequencing; NIPT

Author for correspondence:

Katia Margiotti,

E-mail: katia.margiotti@artemisia.it (c) The Author(s), 2020. Published by Cambridge University Press. This is an Open Access article, distributed under the terms of the Creative Commons Attribution licence (http://creativecommons.org/licenses/by/4.0/), which permits unrestricted re-use, distribution, and reproduction in any medium, provided the original work is properly cited.

\section{CAMBRIDGE} UNIVERSITY PRESS

\title{
Cell-free DNA screening for aneuploidies in 7113 pregnancies: single Italian centre study
}

\author{
Alvaro Mesoraca ${ }^{1}$, Katia Margiotti ${ }^{1}$ (D), Claudio Dello Russo ${ }^{1}$, Anthony Cesta ${ }^{1}$, \\ Antonella Cima ${ }^{1}$, Salvatore Antonio Longo ${ }^{1}$, Maria Antonietta Barone ${ }^{1}$, \\ Antonella Viola ${ }^{1}$, Davide Sparacino ${ }^{1}$ and Claudio Giorlandino ${ }^{1,2}$
}

${ }^{1}$ Human Genetics Lab, Altamedica Main Centre, Viale Liegi 45, 00198 Rome, Italy and ${ }^{2}$ Department of Prenatal Diagnosis, Altamedica, Fetal-Maternal Medical Centre, Viale Liegi 45, 00198 Rome, Italy

\begin{abstract}
Introduction. Non-invasive prenatal testing (NIPT) using cell-free foetal DNA has been widely accepted in recent years for detecting common foetal chromosome aneuploidies, such as trisomies 13, 18 and 21, and sex chromosome aneuploidies. In this study, the practical clinical performance of our foetal DNA testing was evaluated for analysing all chromosome aberrations among 7113 pregnancies in Italy.

Methods. This study was a retrospective analysis of collected NIPT data from the Ion S5 nextgeneration sequencing platform obtained from Altamedica Medical Centre in Rome, Italy. Results. In this study, NIPT showed $100 \%$ sensitivity and $99.9 \%$ specificity for trisomies 13,18 and 21 . Out of the 7113 samples analysed, 74 cases (1\%) were positive by NIPT testing; foetal karyotyping and follow-up results validated 2 trisomy 13 cases, 5 trisomy 18 cases, 58 trisomy 21 cases and 10 sex chromosome aneuploidy cases. There were no false-negative results. Conclusion. In our hands, NIPT had high sensitivity and specificity for common chromosomal aneuploidies such as trisomies 13,18 and 21 .
\end{abstract}

\section{Introduction}

Until recently, prenatal screening for foetal aneuploidies relied on measurement of maternal serum biochemical markers such as serum $\alpha$-fetoprotein (AFP) or $\beta$-human chorionic gonadotropin $(\beta$-hCG) combined with an ultrasound test. The discovery of cell-free foetal DNA (cffDNA) in maternal plasma prompted the development of non-invasive prenatal testing (NIPT), introducing into clinical practice a new approach for the screening of common foetal aneuploidies, reducing unnecessary invasive procedures such us amniocentesis and chorionic villus sampling that may result in miscarriage or intrauterine infection (Odibo et al., 2008). NIPT is based mainly on targeted and whole-genome-based technologies employing nextgeneration sequencing (NGS). These technologies rely on the ability to detect increases in cffDNA arising from the presence of an extra foetal chromosome. The clinical introduction of NIPT NGS has been successfully reported in many clinical validation studies (Sehnert et al., 2011; Norton et al., 2012; Nicolaides et al., 2013; Verweij et al., 2013; Bianchi et al., 2014; Pergament et al., 2014), showing a detection rate of more than $99 \%$, with a foetal fraction of less than $1 \%$ for trisomy 21 (Gil et al., 2014). According to a systematic review and meta-analysis, the pooled sensitivity was $97.4 \%$ for trisomy $13,97.4 \%$ for trisomy 18 and $99.3 \%$ for trisomy 21 (Taylor-Phillips et al., 2016). NIPT clinical services were introduced in Italy in 2012, but a large-scale clinical study is still lacking. The objective of this study was to evaluate the overall clinical performance of our foetal DNA NIPT-NGS-based methodology in detecting trisomies 13,18 and 21 and sex chromosome aneuploidies (SCAs) in a cohort of 7113 samples.

\section{Materials and methods}

\subsection{Subjects}

The study includes the retrospective investigation of 7113 pregnant women who were admitted at Altamedica Medical Centre (Rome, Italy) and underwent foetal cffDNA screening tests between January 2018 and March 2019. The present study was approved by the local Ethics Committee of Artemisia SPA and all participating women provided their written informed consent. In this cohort, confirmation feedback was available for positive sample results.

\subsection{Sample collection and NIPT analysis}

In total, 7113 cases were tested using the Ion S5 NGS (ThermoFisher Scientific, Waltham, MA, USA) platform. The features of the cases and their indications for NIPT are summarized in Table 1. 
Table 1. Patient characteristics of 7113 pregnancies undergoing non-invasive prenatal testing (NIPT) for chromosomal aneuploidy.

\begin{tabular}{ll}
\hline Clinical characteristics & \multicolumn{1}{c}{$\begin{array}{c}\text { Mean value } \\
\text { (range) }\end{array}$} \\
\hline Maternal age & $33.4(20-50)$ \\
\hline Body mass index $\left(\mathrm{kg} / \mathrm{m}^{2}\right)$ & $23.2(14.5-43.5)$ \\
\hline Gestational age (weeks) & $16.5(10-26)$ \\
\hline Indications for NIPT & Ratios (\%) \\
\hline Maternal serum screening result & $26.3 \%$ \\
\hline Advanced maternal age $\geq 35$ years & $41.2 \%$ \\
\hline $\begin{array}{l}\text { Others (family history, in vitro fertilization } \\
\text { pregnancy, decision of perinatology council, etc.) }\end{array}$ & $32.5 \%$ \\
\hline
\end{tabular}

The following protocol was used: blood samples were collected in Streck Cell-Free DNA BCT tubes (Streck, La Vista, NE, USA) and sample were centrifuged on the same day. Approximately 3-4 $\mathrm{mL}$ of plasma was isolated and stored at $-80^{\circ} \mathrm{C}$ until cffDNA extraction. The cffDNA was extracted using the Qiasymphony DSP Virus or Qiasymphony Circulating DNA Kit (Qiagen, Valencia, CA, USA). The DNA concentration was determined using the Agilent Technologies 4200 TapStation System (Agilent Technologies, Santa Clara, CA, USA) and the High Sensitivity D1000 ScreenTape System (Agilent Technologies). The acceptable cffDNA range for processing the sample was between 80 and 120 pg/ $\mu \mathrm{L}$. DNA libraries were prepared using the Ion Ampliseq Kit according to the manufacturer's instructions (ThermoFisher Scientific). The resulting libraries were sequenced using the Ion S5 system. The results of the tests were provided to patients by post-test counselling; invasive diagnosis was offered for positive reports. Generally, invasive testing methods such as quantitative fluorescent polymerase chain reaction and karyotyping by amniocentesis were selected in order to confirm the results.

\subsection{Data analysis}

Sequencing data were analysed using a proprietary algorithm developed by Life\&Soft Company (Plessis-Robinson, France). Briefly, the detection of foetal aneuploidies is based on a combination of high-quality alignments against the human genome and read counts to identify chromosomal gains. A first normalization is performed based on GC percentage to calculate a high-quality $\mathrm{Z}$-score. A second normalization is performed in each sample to compute another $\mathrm{z}$-score based on a chromosome not involved in the aneuploidy detection. The presence of a foetal aneuploidy for all 24 chromosomes is assessed using the values of the two $\mathrm{Z}$-score calculations. A lack of a result in a sample was attributed to an insufficient $(<4 \%)$ fraction of cffDNA or failure to pass the quality control measures. Based on these results, chromosomal aneuploidy was determined in the foetus. The sensitivity, specificity and positive predictive value (PPV) were calculated using the VassarStats online calculator (http://www.vassarstats.net).

\section{Results and discussion}

The objective of this study was to evaluate the performance of our foetal DNA test in detecting trisomies 13, 18, and 21 and SCAs in a cohort of 7113 high-risk or intermediate-risk pregnancies arriving in our laboratory between January 2018 and March 2019. The median gestational age in this testing cohort was 16.5 weeks (Table 1). The median maternal age was 33.4 years. The median body mass index was $23.2 \mathrm{~kg} / \mathrm{m}^{2}$. The median foetal fraction of reported samples was $7.3 \%$. In this cohort, twin pregnancy samples represented $0.8 \%$ of all pregnancies tested. The median time for reporting was 5 business days. Repeat samples were reported in $1.6 \%(119 / 7113)$ of the sample cohort, and these were due to insufficient foetal fraction (below a pre-specified threshold of $4 \%$ ), low reads and discordant sex. In this cohort, the overall frequency for autosomal aneuploidies was $0.9 \%$ for trisomies 13,18 and 21 collectively, while the frequency of reported SCAs was $0.14 \%$ (Table 2). In order to assess the accuracy of the positive NIPT results, follow-up based on confirmatory testing using amniocentesis for abnormal samples is recommended. Follow-up confirmation results were available for 3 samples with trisomy 13, 7 samples with trisomy 18, 62 samples with trisomy 21 and 13 samples with SCAs, reported as 'Confirmed' in Table 2. Data on pregnancy outcome were missing for five cases, in particular two for trisomy 18, one for monosomy $\mathrm{X}$, one for trisomy X and one for 47, XYY karyotypes, all reported as 'Unconfirmed' in Table 2. Missing data were due to loss of contact or because the women declined follow-up. Based on the confirmatory follow-up, the PPVs indicating the probability that a foetus with a positive NIPT test truly has the genetic disorder were $66.7 \%$ for trisomy $13,71.4 \%$ for trisomy 18 and $93.5 \%$ for trisomy 21 (Table 2). Since all negative NIPT cases were found to be negative based on this outcome, we assume the mutation detection rate of our test to be close to $100 \%$. Follow-up results for SCAs were obtained for nine samples with monosomy $\mathrm{X}$ (Turner syndrome), two samples with trisomy $\mathrm{X}$, two samples with 47, XXY (Klinefelter syndrome) and no samples with 47, XYY. Estimated PPVs were $66.7 \%$ for monosomy X and $100 \%$ for trisomy X and 47, XXY (Table 2). Among 7113 pregnant women who underwent NIPT analysis, we diagnosed 90 cases with abnormal NIPT results. Of these, 75 cases were confirmed to be true-positive results with PPVs of $66.7 \%, 71.4 \%$ and 93.5\% for trisomies 13, 18 and 21, respectively, and PPVs of $66.7 \%$ for monosomy X and $100 \%$ for trisomy X and $47, \mathrm{XXY}$, while 5 cases could not be considered further due to a lack of communication with the patients (Table 2).

cffDNA screening for aneuploidies by NGS-based methodologies has been widely used for trisomies 13,18 and 21 in recent years, but clinical studies on its efficacy at single centres are lacking. Foetal DNA testing is a NIPT test with high performance that is able to detect common autosomal trisomies and SCAs such as monosomy X, 47, XXY, trisomy X and 47, XYY. Our test results were SCA positive in 16 samples, and 10 samples were validated by karyotyping, showing an overall true-positive rate of $63 \%$. This study has a number of limitations for SCA analysis. First, due to the low incidence of SCAs in the general population (1/400 new-borns) more pregnancies must be evaluated in order to better investigate and define the accuracy of this test for the detection of SCA syndromes. Secondly, since new-borns with SCA syndromes can appear phenotypically normal, the negative predictive value could not be calculated, and so caution needs to be expressed in these types of studies, unless all neonates undergo karyotyping analysis.

Nevertheless, in our population, the PPV was higher than or similar to that reported by others (Yao et al., 2014; Bianchi et al., 2015). Petersen et al. (2017) found PPVs of 26\% for monosomy X, $50 \%$ for trisomy $\mathrm{X}$ and $86 \%$ for $47, \mathrm{XXY}$.

Our prospective study collected 7113 clinical samples and evaluated the NIPT strength by analysing the cytogenetics or 
Table 2. Foetal DNA clinical performance.

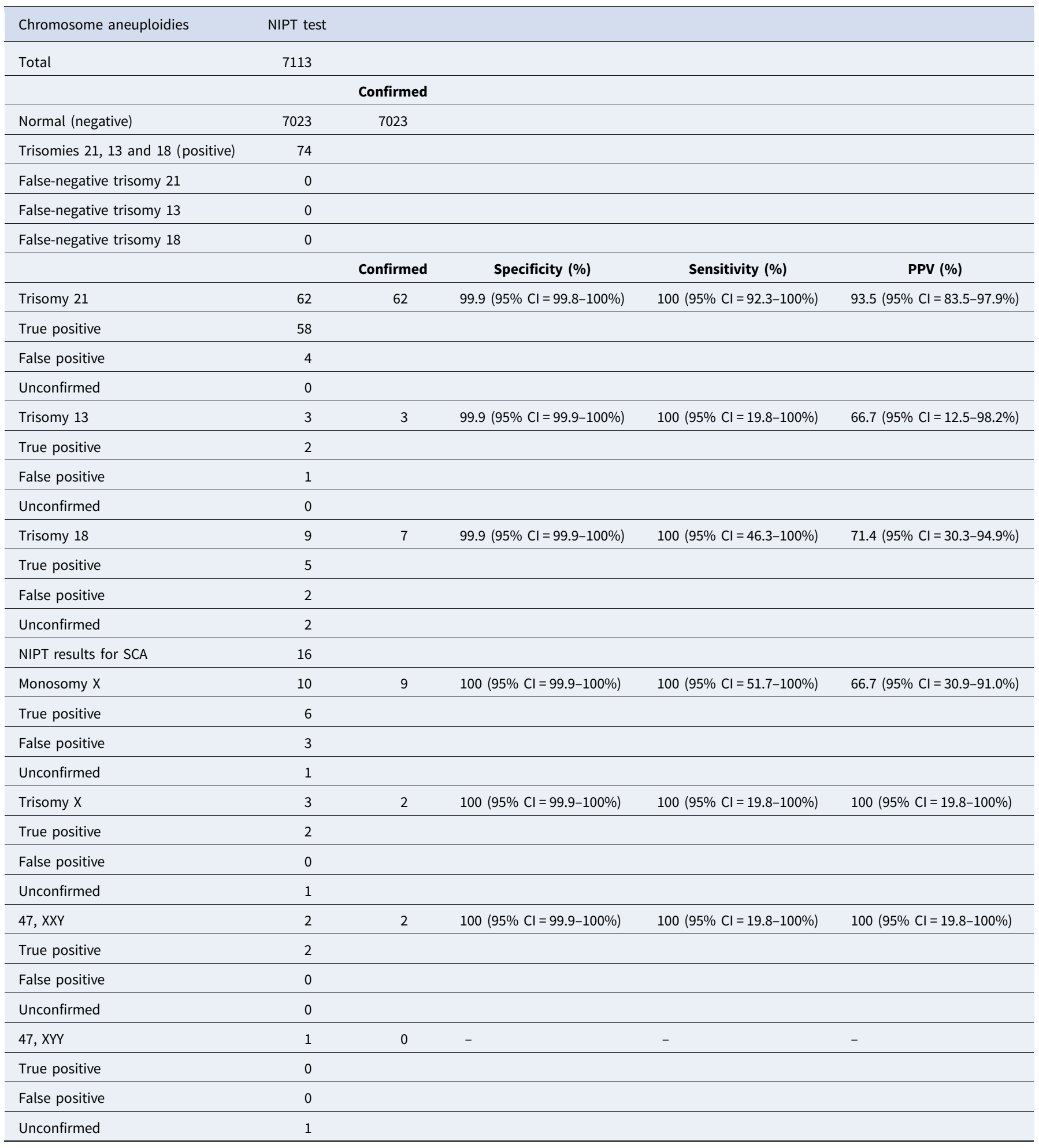

$\mathrm{Cl}=$ confidence interval; NIPT = non-invasive prenatal testing; PPV = positive predictive value; SCA = sex chromosome aneuploidy.

phenotypic outcomes. In conclusion, our study represents a clinical experience of NIPT in Italy where prenatal screening by this method is used predominantly as a secondary screening test. We have shown that the performance of NIPT in detecting trisomies 13,18 and 21 was maintained at a high level, comparable to that of a recently validated clinical study (Kypri et al., 2019). This study offers further proof of the high degree of accuracy of NGS-based NIPT analysis compared to conventional screening methods.

\section{Author contributions.}

All authors have materially participated in the study and manuscript preparation. CDR, $\mathrm{AC}, \mathrm{SAL}$ and $\mathrm{MAB}$ carried out all of the molecular genetic analysis and participated in the design of the work; AC, AV, DS and KM collected all clinical data and participated 
in conceiving the manuscript; AM and CG participated in conceiving the study and drafted and revised the manuscript. All authors have approved the final article.

Conflicts of interest. AM, KM, CDR, AC, AC, SAL, MAB, AV and DS are employed by Altamedica Medical Centre of Rome and CG is the scientific director of Altamedica Medical Centre.

Financial support. No funding was received to support this study.

\section{References}

Bianchi D.W., Parker R.L., Wentworth J. et al. (2014). DNA sequencing versus standard prenatal aneuploidy screening. New England Journal of Medicine 370(9), 799-808.

Bianchi D.W., Parsa S., Bhatt S. et al. (2015). Fetal sex chromosome testing by maternal plasma DNA sequencing: clinical laboratory experience and biology. Obstetrics and Gynecology 125(2), 375-382.

Gil M.M., Akolekar R., Quezada M.S., Bregant B. and Nicolaides K.H. (2014). Analysis of cell-free DNA in maternal blood in screening for aneuploidies: meta-analysis. Fetal Diagnosis and Therapy 35(3), 156-173.

Kypri E., Ioannides M., Touvana E. et al. (2019). Non-invasive prenatal testing of fetal chromosomal aneuploidies: validation and clinical performance of the veracity test. Molecular Cytogenetics 12(1), 34.

Nicolaides K.H., Syngelaki A., Gil M., Atanasova V. and Markova D. (2013). Validation of targeted sequencing of single-nucleotide polymorphisms for non-invasive prenatal detection of aneuploidy of chromosomes 13, 18, 21, $\mathrm{X}$, and Y. Prenatal Diagnosis 33(6), 575-579.

Norton M.E., Brar H., Weiss J. et al. (2012). Non-Invasive Chromosomal Evaluation (NICE) study: results of a multicenter prospective cohort study for detection of fetal trisomy 21 and trisomy 18. American Journal of Obstetrics and Gynecology 207(2), 137.e1-137.e8.

Odibo A.O., Gray D.L., Dicke J.M., Stamilio D.M., Macones G.A. and Crane J.P. (2008). Revisiting the fetal loss rate after second-trimester genetic amniocentesis: a single center's 16-year experience. Obstetrics and Gynecology 111(3), 589-595.

Pergament E., Cuckle H., Zimmermann B. et al. (2014). Single-nucleotide polymorphism-based noninvasive prenatal screening in a high-risk and low-risk cohort. Obstetrics and Gynecology 124(2 Pt 1), 210-218.

Petersen A.K., Cheung S.W., Smith J.L. et al. (2017). Positive predictive value estimates for cell-free noninvasive prenatal screening from data of a large referral genetic diagnostic laboratory. American Journal of Obstetrics and Gynecology 217(6), 691.e1-691.e6.

Sehnert A.J, Rhees B., Comstock D. et al. (2011). Optimal detection of fetal chromosomal abnormalities by massively parallel DNA sequencing of cell-free fetal DNA from maternal blood. Clinical Chemistry 57(7), 1042-1049.

Taylor-Phillips S., Freeman K., Geppert J. et al. (2016). Open accuracy of non-invasive prenatal testing using cell-free DNA for detection of Down, Edwards and Patau syndromes: a systematic review and meta-analysis. BMJ Open 6(1), e010002.

Verweij E.J., Jacobsson B., van Scheltema P.A. et al. (2013). European Non-Invasive Trisomy Evaluation (EU-NITE) study: a multicenter prospective cohort study for non-invasive fetal trisomy 21 testing. Prenatal Diagnosis 33(10), 996-1001.

Yao H., Jiang F., Hu H. et al. (2014). Detection of fetal sex chromosome aneuploidy by massively parallel sequencing of maternal plasma DNA: initial experience in a Chinese hospital. Ultrasound in Obstetrics and Gynecology 44(1), 17-24. 